\title{
Reduction of Neuroinflammation in Epilepsy by Using Induced Pluripotent Stem (iPS) Cells-Derived
}

\author{
Astrocytes
}

\author{
Azadeh Sajadian', Maryam Jafarian ${ }^{1,2}$, Babak Khodaie ${ }^{1}$, Shahin Mohammad Sadeghi ${ }^{3}$, Amir Ghaemi ${ }^{4 *}$ \\ ${ }^{1}$ Shefa Neuroscience Research Center, Khatam Alanbia Hospital, Tehran, Iran. \\ ${ }^{2}$ School of Advanced Technologies in Medicine, Tehran University of Medical Sciences, Tehran, Iran. \\ ${ }^{3}$ Department of Plastic and Reconstructive Surgery, Shahid Beheshti University of Medical Sciences, Tehran, Iran. \\ ${ }^{4}$ Department of Microbiology, School of Medicine, Golestan University of Medical Sciences, Gorgan, Iran.
}

\section{A BSTRACT}

Introduction: The ability to induce pluripotency in somatic cells by reprogramming factors offers new opportunities for drug discovery and cell therapy. Induced pluripotent stem cells have the potential to differentiate to various cell types, such as neural and glial cells. Astrocytes, the major glial cells of the central nervous system, play an important role in the function of the brain by regulating of extracellular ions and neurotransmitters, feeding and protection of neurons as well as modulating the activity of microglia. Microglia over-activation can be resulted in brain inflammation with subsequent susceptibility to epileptic seizures. Hypothesis: For many years, embryonic Stem cell transplantation has been examined to prevent seizure attacks in epilepsy. These studies have indicated that adult cells from patient have the ability to be transformed to embryonic stage and convert to a pluripotent stem cell by using some Transcription factors (such as Oct4, Sox2, Nanog, Rex1, Klf, c-Myc and LIN28). Accordingly, fibroblasts from an epileptic have also been reprogrammed to embryonic stage. The resulting iPS cells are isogenic to patient and are able to transform to neurons or glia in a suitable culture condition. Previous studies on ES cell therapy have focused more on neurons than astrocytes. Astrocytes, by secretion of glial cell-derived neurotrophic factor, not only regulate the different microglial activities, such as proliferation, migration and cell adhesion, but can also reduce destructive effects of microglia. Conclusion: In this hypothesis, we suggest a reprogramming system for generating functional astrocyte from human pluripotent stem cell in the presence of neural growth factors. We hypothesize that these cells might reduce neuroinflammation induced by microglia and subsequent susceptibility to seizure. The reprogrammed cells could be used in cell replacement therapy of epilepsy.

\section{Key words:}

1. Astrocytes

2.Seizures

3.Epilepsy

4. Microglia

5.Induced Pluripotent

Stem Cells.

\section{*Corresponding Author: Amir Ghaemi}

E-mail:ghaem_amir@yahoo.com 


\title{
كاهش التهاب عصبى در صرع با استفاده از آستروسيتهاى مشتق شده از سلولهاى بنيادى بر توان القايى
}

\author{
آزاده سجاديان'، مريم جعفريان ‘'، بابك خدائى'، شاهين محمدصادقى"، امير قائمى ": \\ 'مركز تحقيقات علوم اعصاب شفا، بيمارستان خاتم الانبياء، تهران، ايران.

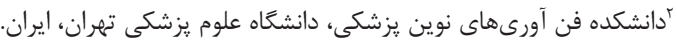

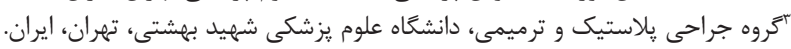

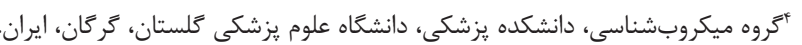

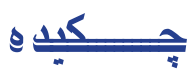

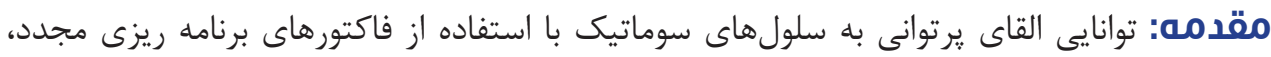

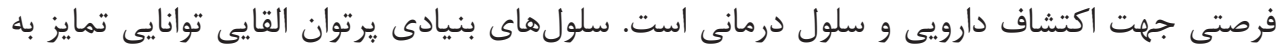

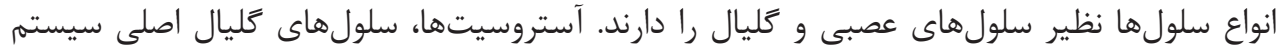

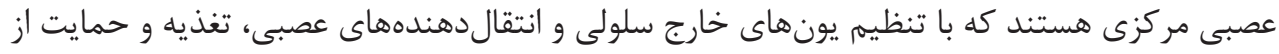

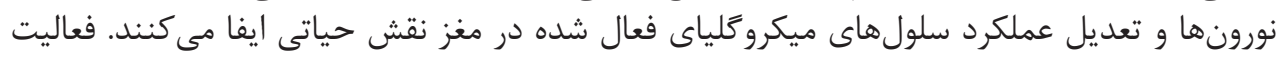

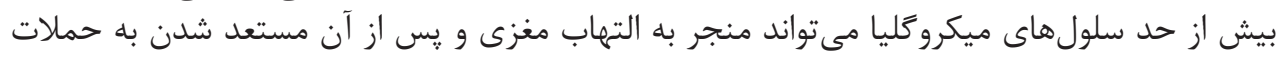

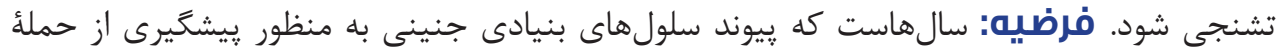

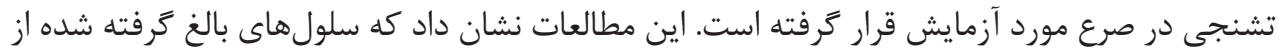

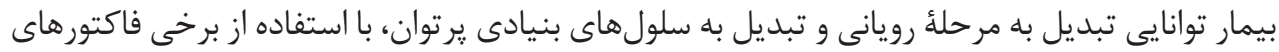

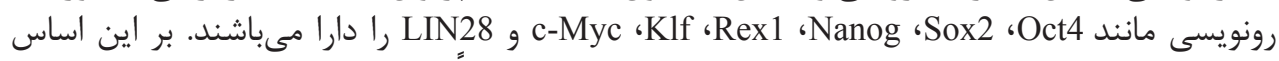

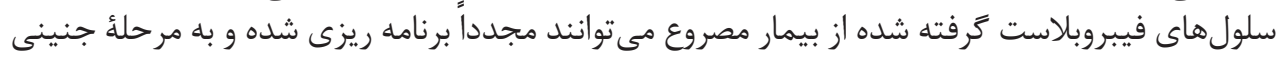

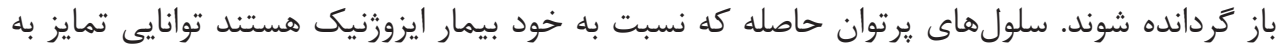

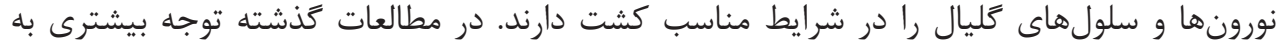

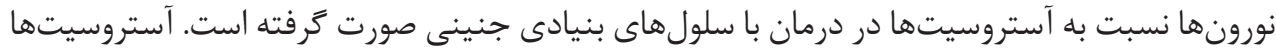

كليد وازهها: ا I. آستروسيت

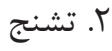

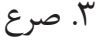

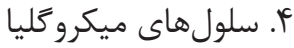

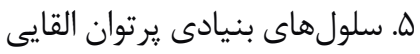

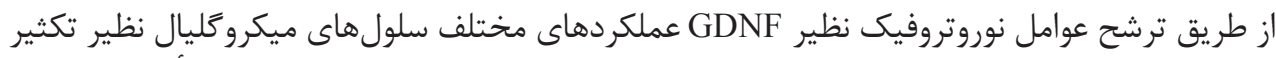

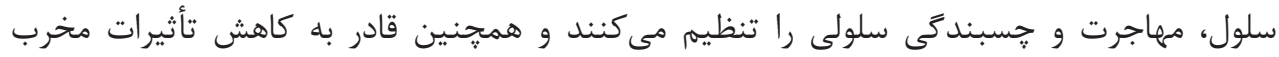

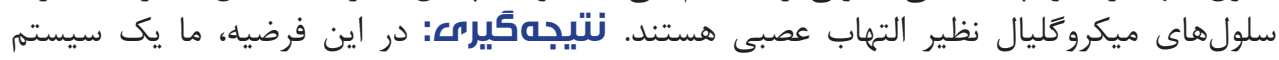

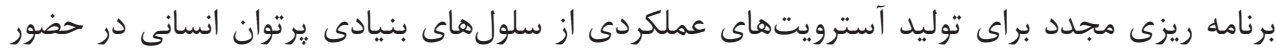

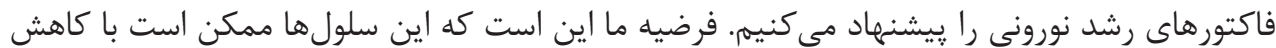

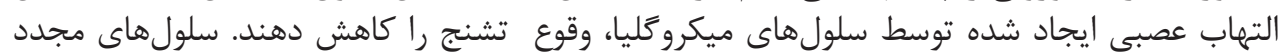

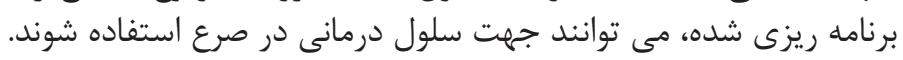

$$
\text { " نويسنده مسئول: امير قائمى آدرس الكترونيكى: ghaem_amir@yahoo.com }
$$


يروتئين منجر به عدم توانايى تشكيل فيلامنتهاى حد واسط

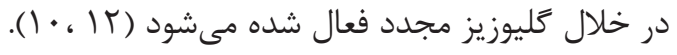

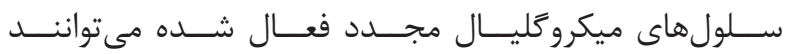

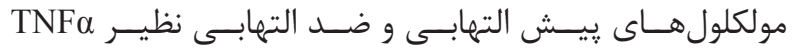

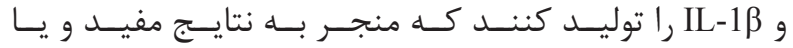

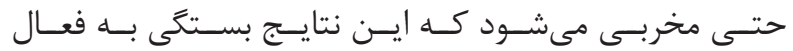

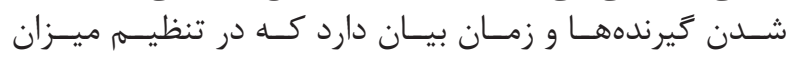

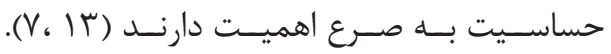

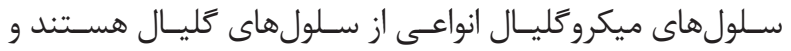

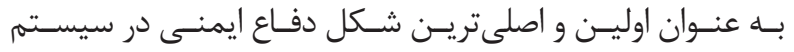

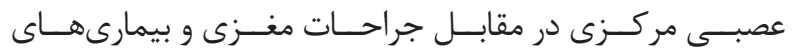

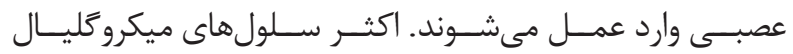

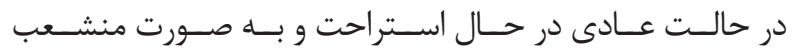

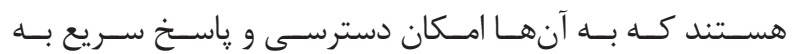

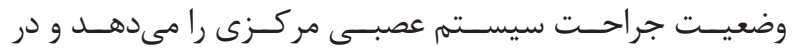

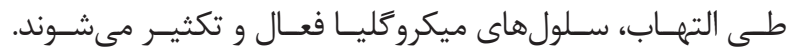

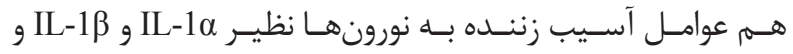

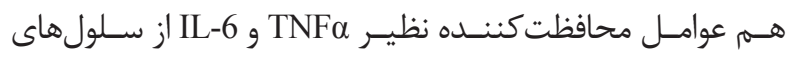

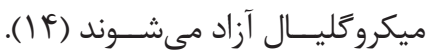

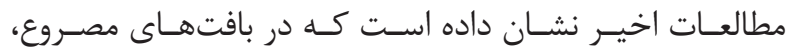

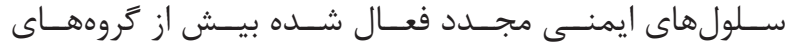

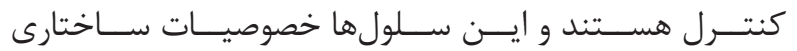

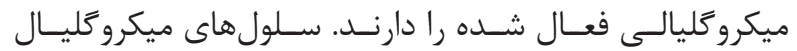

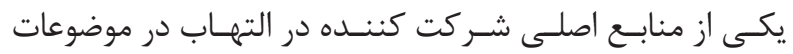

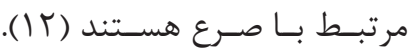

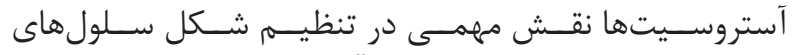

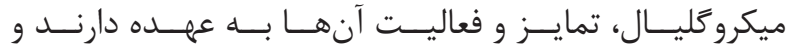

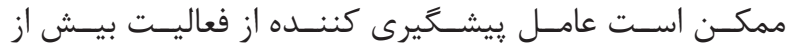

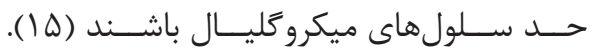

$$
\text { سلول درمانى در صرع }
$$

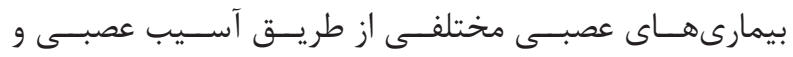

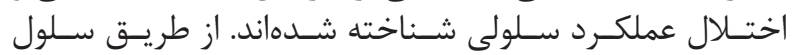

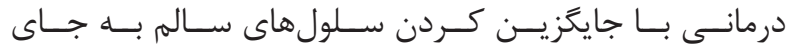

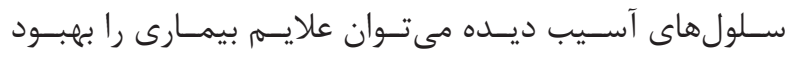

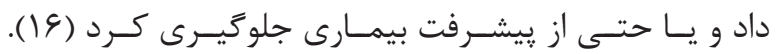

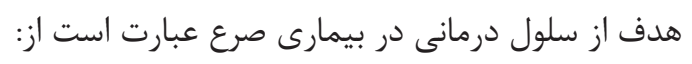

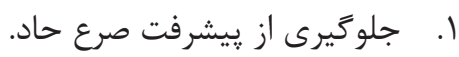

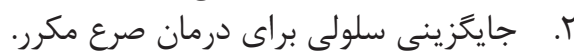

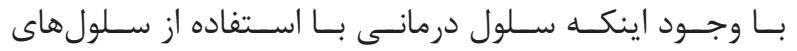

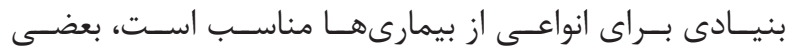

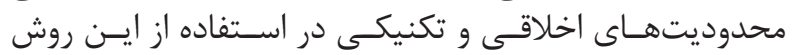

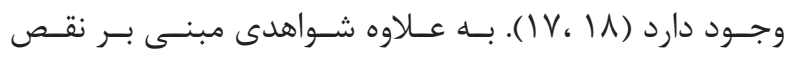

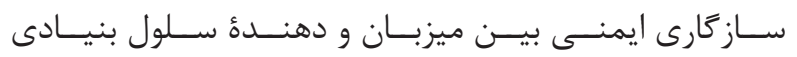

\section{${ }^{1}$ Epilepsy}

${ }^{2}$ Temporal Lobe Epilepsy

${ }^{3}$ Hippocamp

${ }^{4}$ Neuroinflammation

${ }^{5}$ Activated Glial Cells

${ }^{6}$ Intermediated filaments
صرع كَيجًاهى

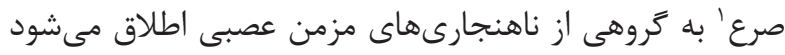

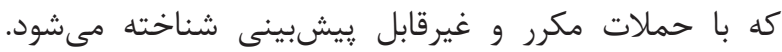

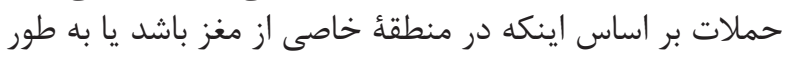

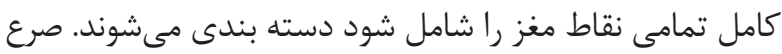

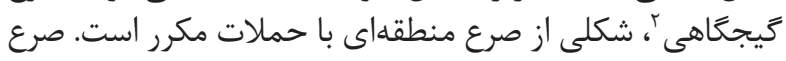

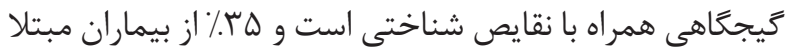

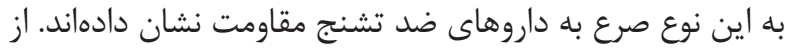

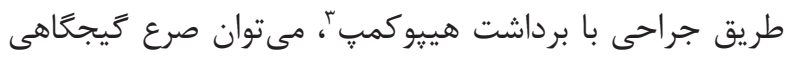

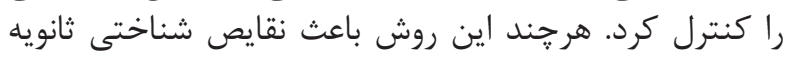

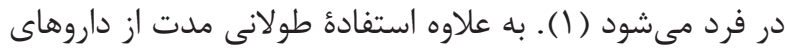

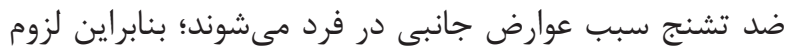

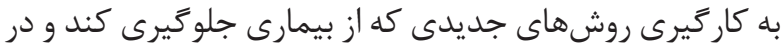

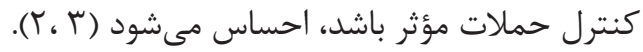

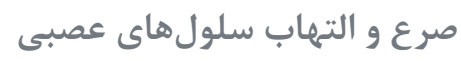

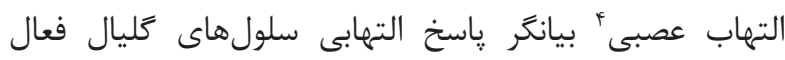

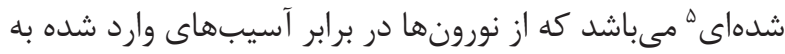

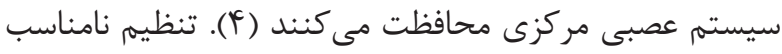

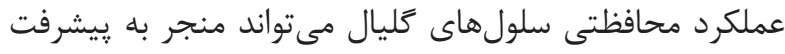

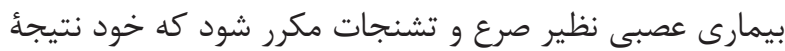

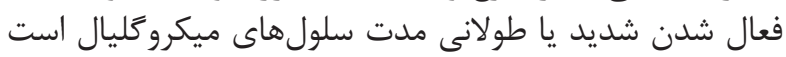

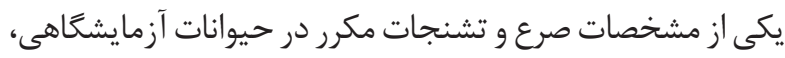

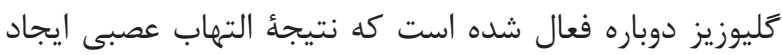

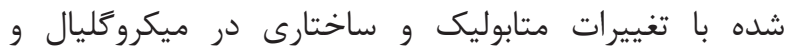
آستروسيتها است. از نظر ساختارى تغييرات ايجاد شده در آستروسيتهاى مجاى مجدد

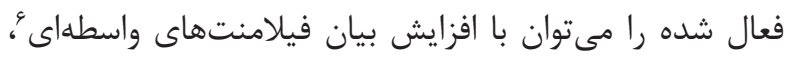

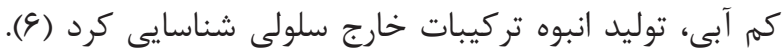

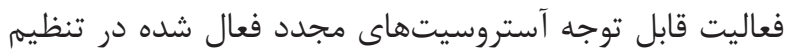

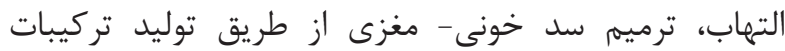

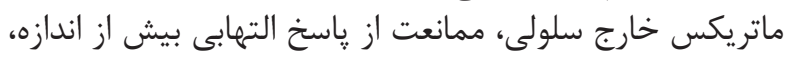

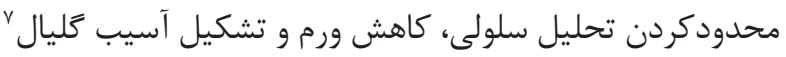

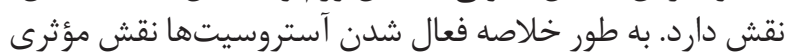

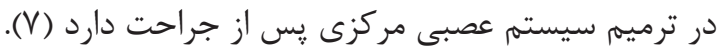
يس از جراحت مغز، تجمع دو نوع از فيلامنتهاى واسطهاى

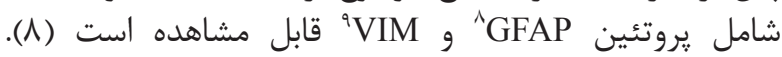

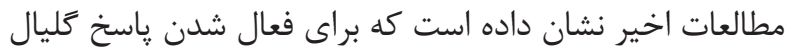

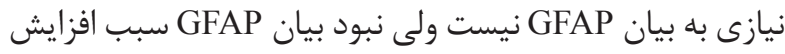

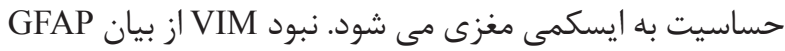

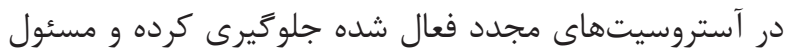

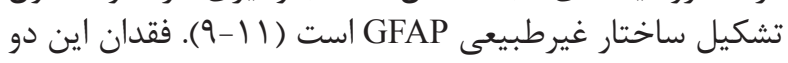

${ }^{7}$ Glial scar

${ }^{8}$ Glial Fibrillary Acidic Protein

${ }^{9}$ Vimentin 
از طريـق روشهــاى مهندسـى زنتيـك، زنهــاى آسـيبديده

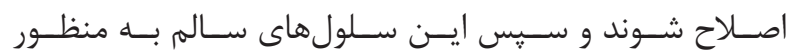

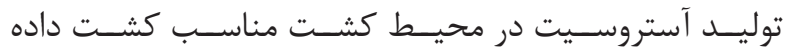

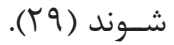

آستروسيت : يك انتخاب ويثُه براى سلول درمانى

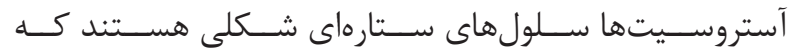

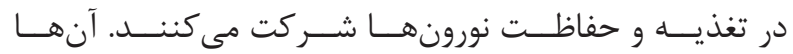

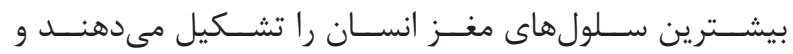

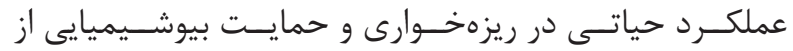

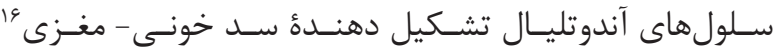

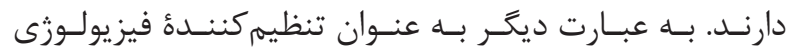

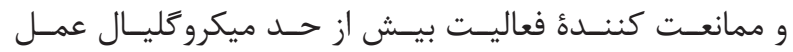

مى كننـــد (V)

بــه طــور خلاصـهـ عملكردهــاى ويـزره آستروســيتها در مغــز

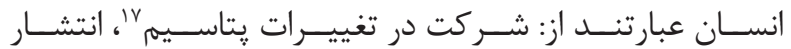

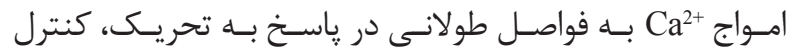

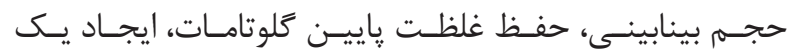

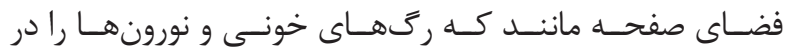

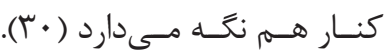

شـواهدى مبنــى بــر افزايـش علايسم شـيميايـى و از بيـن رفتـن

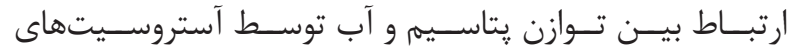

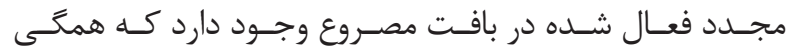

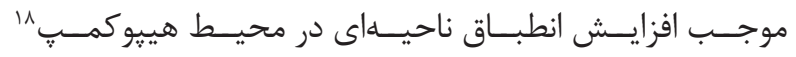

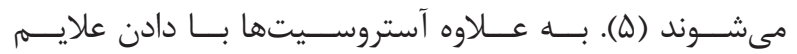

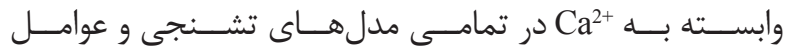

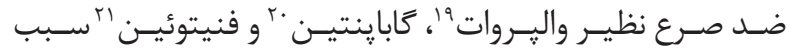

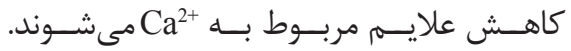

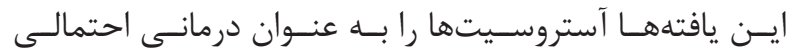

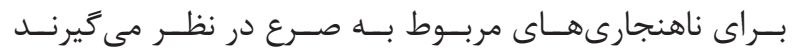

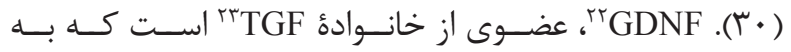

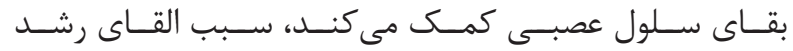

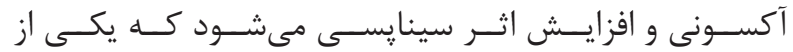

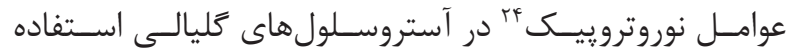

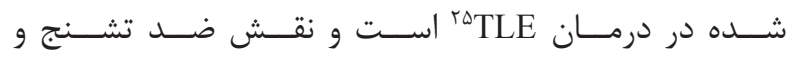

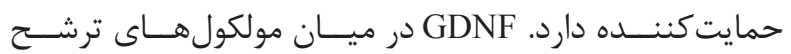

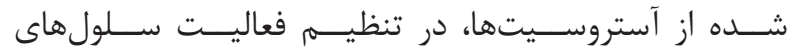

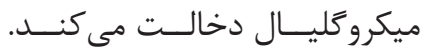

در مدلهـاى آزمايشـعاهى TLE، ممانعـت از صـرع بـا انتقـال

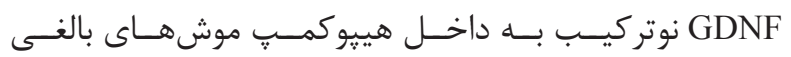

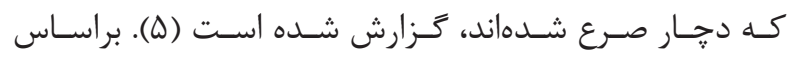

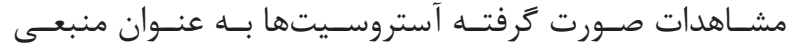

${ }^{10}$ Embryonic Stem Cells

${ }^{11}$ Induced Pluripotent Stem Cells

${ }^{12}$ Self- Renew

${ }^{13}$ Pluripotency

${ }^{14}$ Somatic cell transfer

${ }^{15}$ Reprogramming through cell extract

${ }^{16}$ Blood Brain Barrier

${ }_{17}$ Potassium buffering

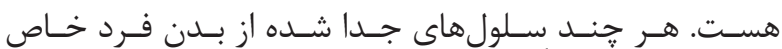

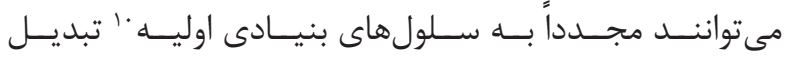

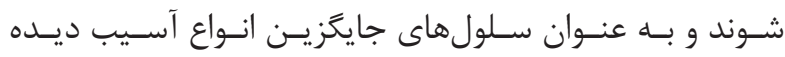

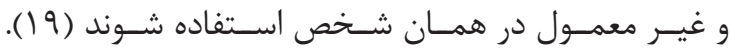

بــا خلـق سـلول هاى بنيــادى يرتــوان القايـى " (iPS Cells)

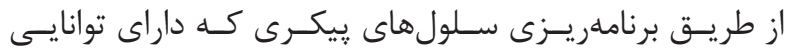

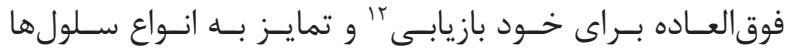

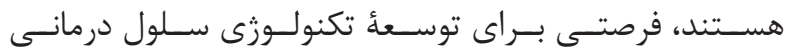

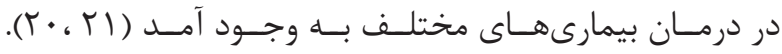

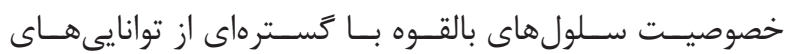

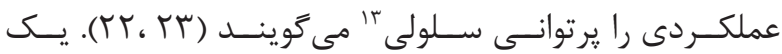

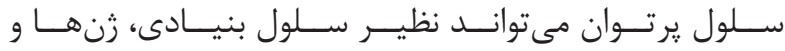

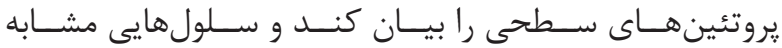

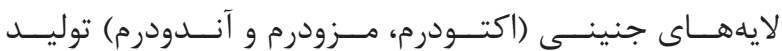

كنــد (T)

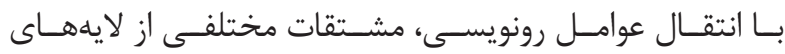

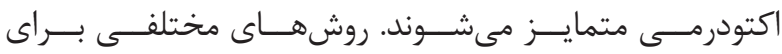

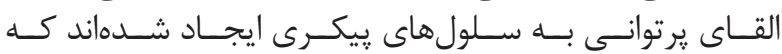

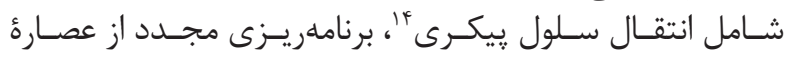

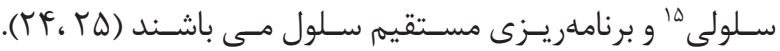

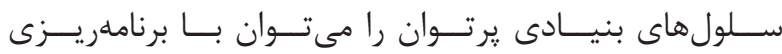

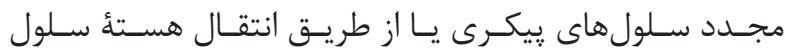

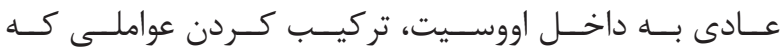

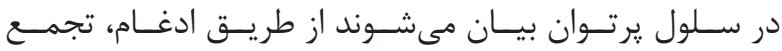

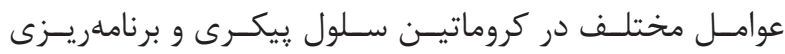

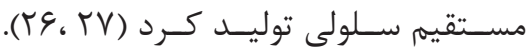

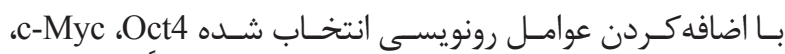

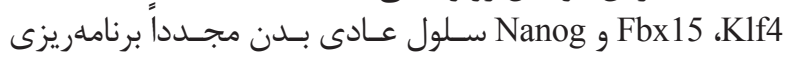

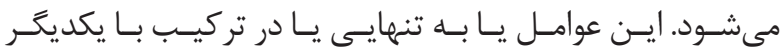

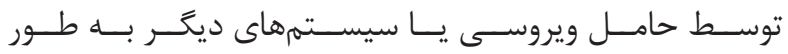

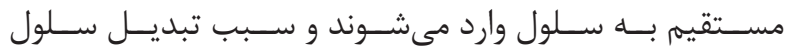

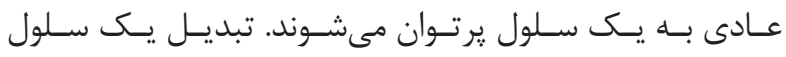

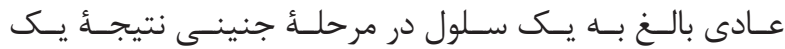

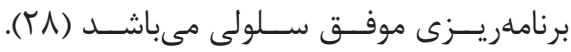

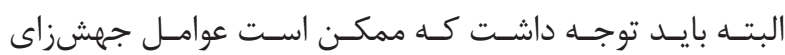

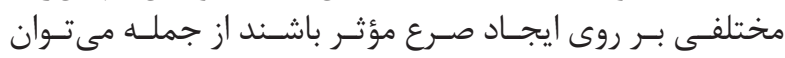

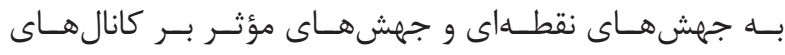

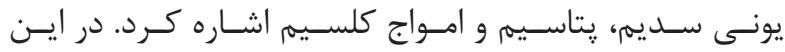

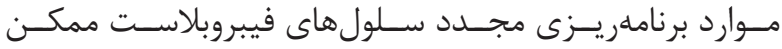

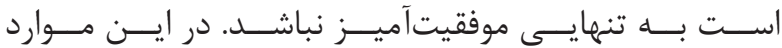

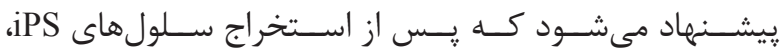

\footnotetext{
${ }^{18}$ Hippocampal microcircuit

${ }^{19}$ Valproate

${ }^{20}$ Gabapentin

${ }^{21}$ Phenytoin

${ }^{22}$ Glial cell line-derived neurotrophic factor (GDNF)

${ }^{23}$ Transforming Growth Factor

${ }^{24}$ Neurotropic

${ }^{25}$ Temporal Lobe Epilepsy
} 


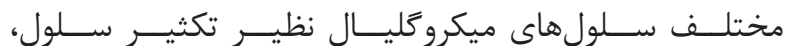

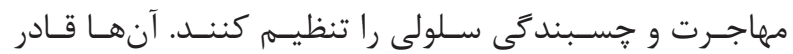

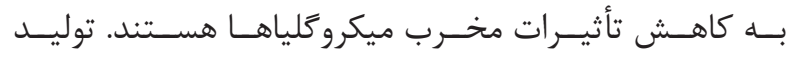

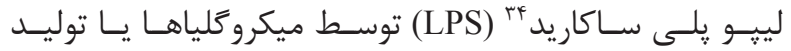

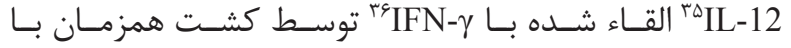

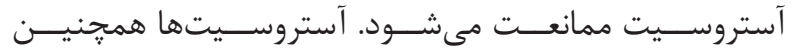

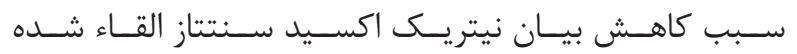

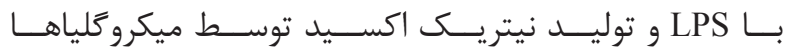

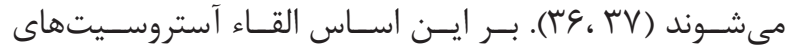

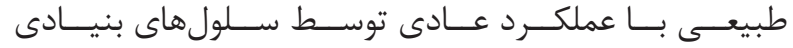

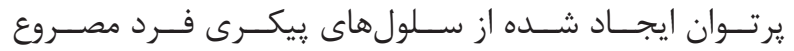

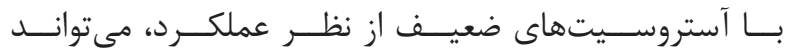

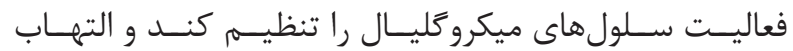

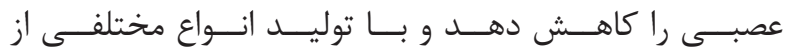

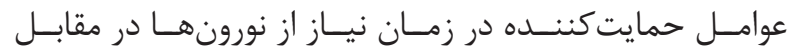

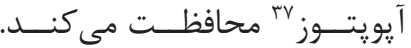

آزمودن فرضيةُ مورد نظر

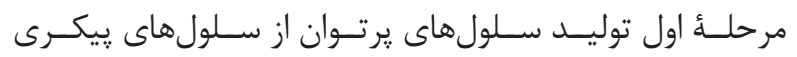

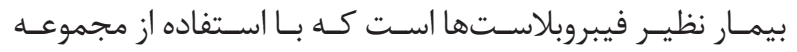

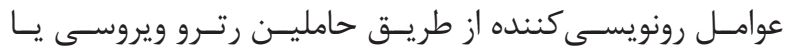

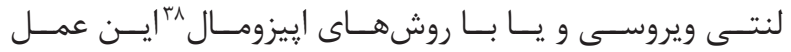

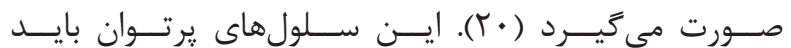

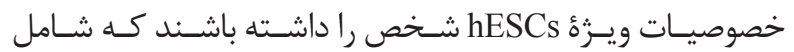

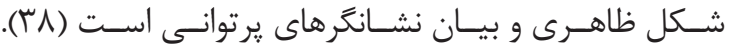

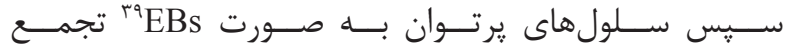

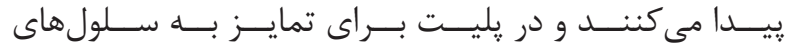

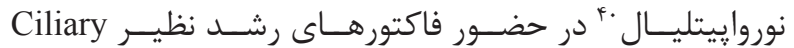
Basic Leukemia inhibitory factor ،neurotropic factor

fibroblast growth factor

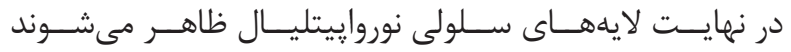

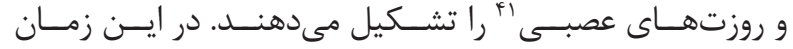

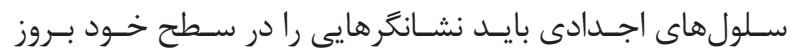

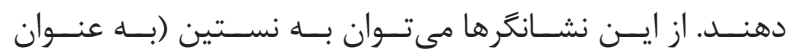

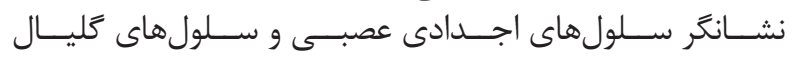

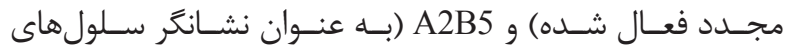

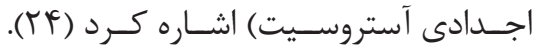

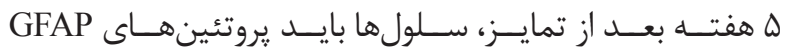

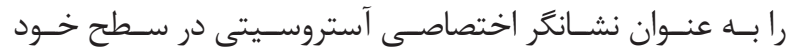

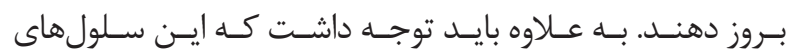

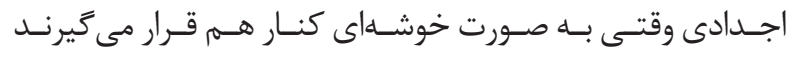

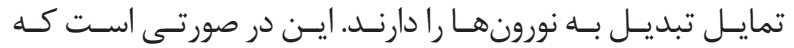

${ }^{26}$ Mediators

${ }^{27}$ Human Embryonic Stem Cells

${ }^{28}$ Somatic cell

${ }^{29}$ Line

${ }^{30}$ Isogenic

${ }^{31}$ Astrocytic

${ }^{32}$ GABA ergic

${ }^{33}$ Glutamatergic

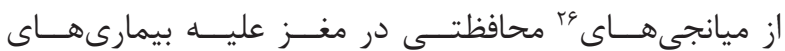

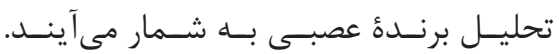

فرضيه

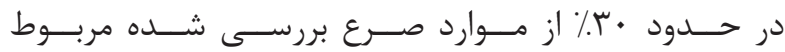

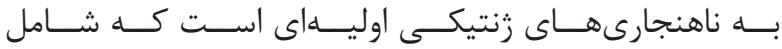

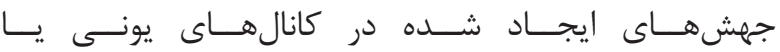

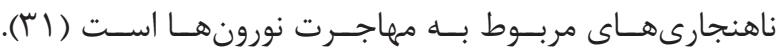

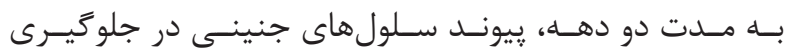

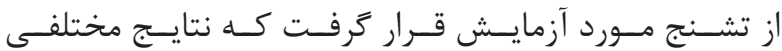

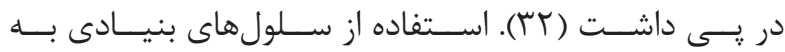

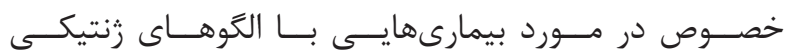

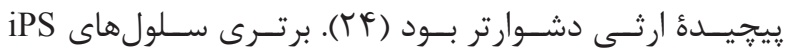

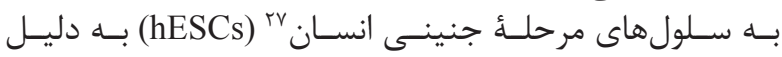

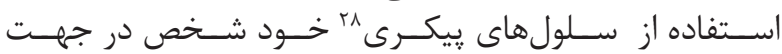

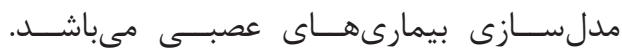

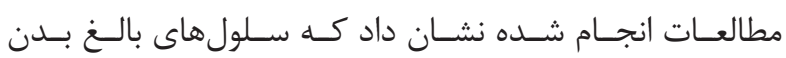

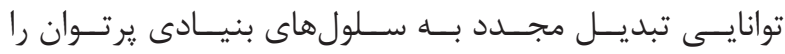

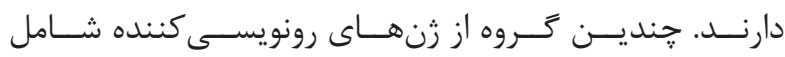
LIN28, c-Myc ،Klf4 ،Rex1 ،Nanog ،Sox2 ،Oct4

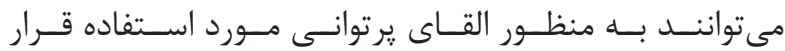

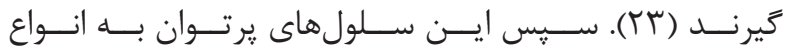

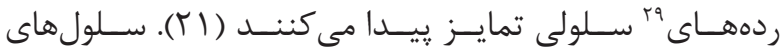

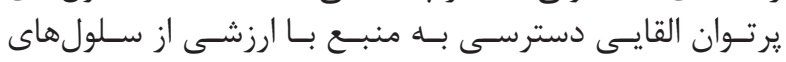

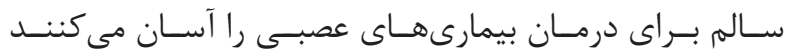

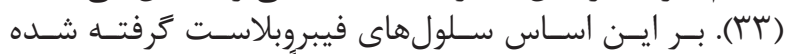

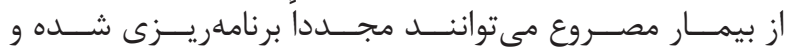

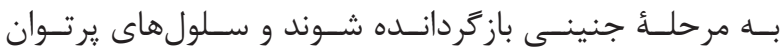

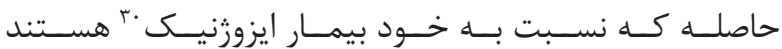

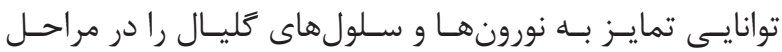

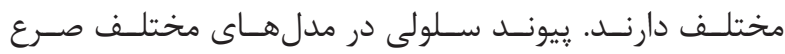

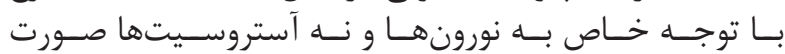

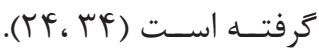

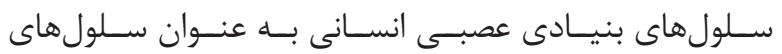

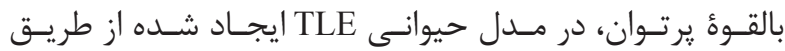

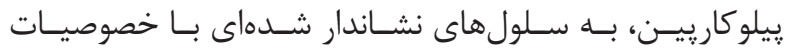

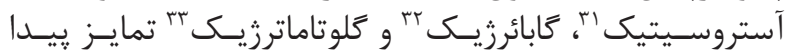

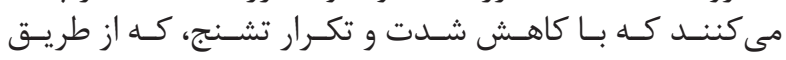

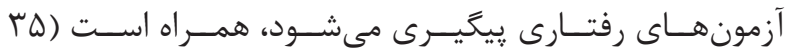
(rr.

از طرفـى مشـخص شـد كـهـ آستروسـيتها از طريـق ترشـح

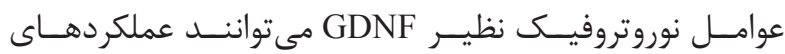

\footnotetext{
${ }^{34}$ Lipopolysaccharide

${ }^{35}$ Interleukin 12

${ }^{36}$ Interferon gamma

${ }^{37}$ Apoptosis

${ }^{38}$ Nonintegrating episomal

${ }^{39}$ Embryoid Bodies

${ }^{40}$ Neuro-Epithelial (NE)

${ }^{41}$ Typical neural rossete
} 


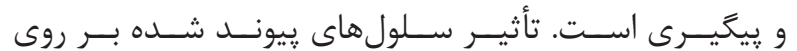

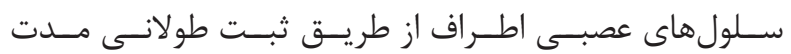

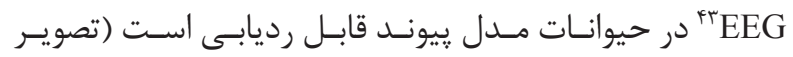

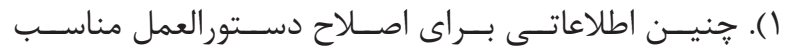

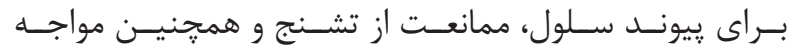

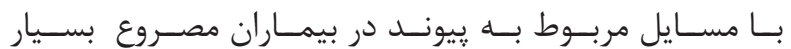

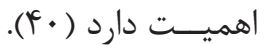

$$
\text { شرح فرضية مورد نظر }
$$

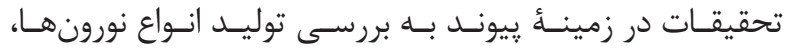

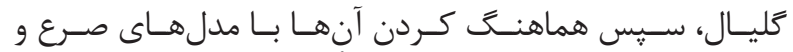

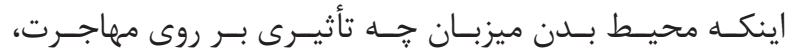

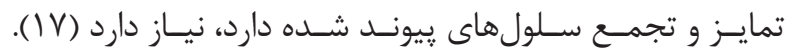

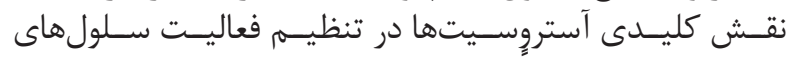

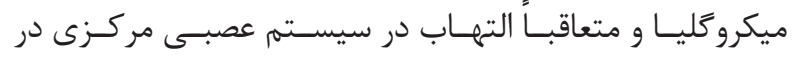

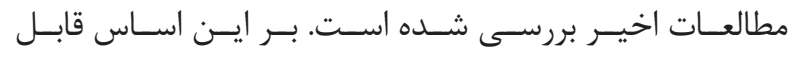

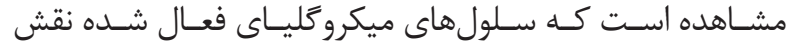

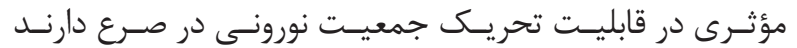

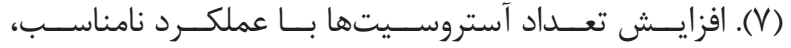

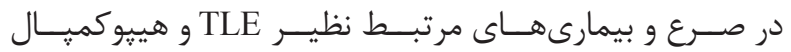

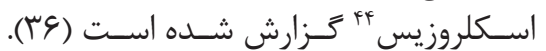

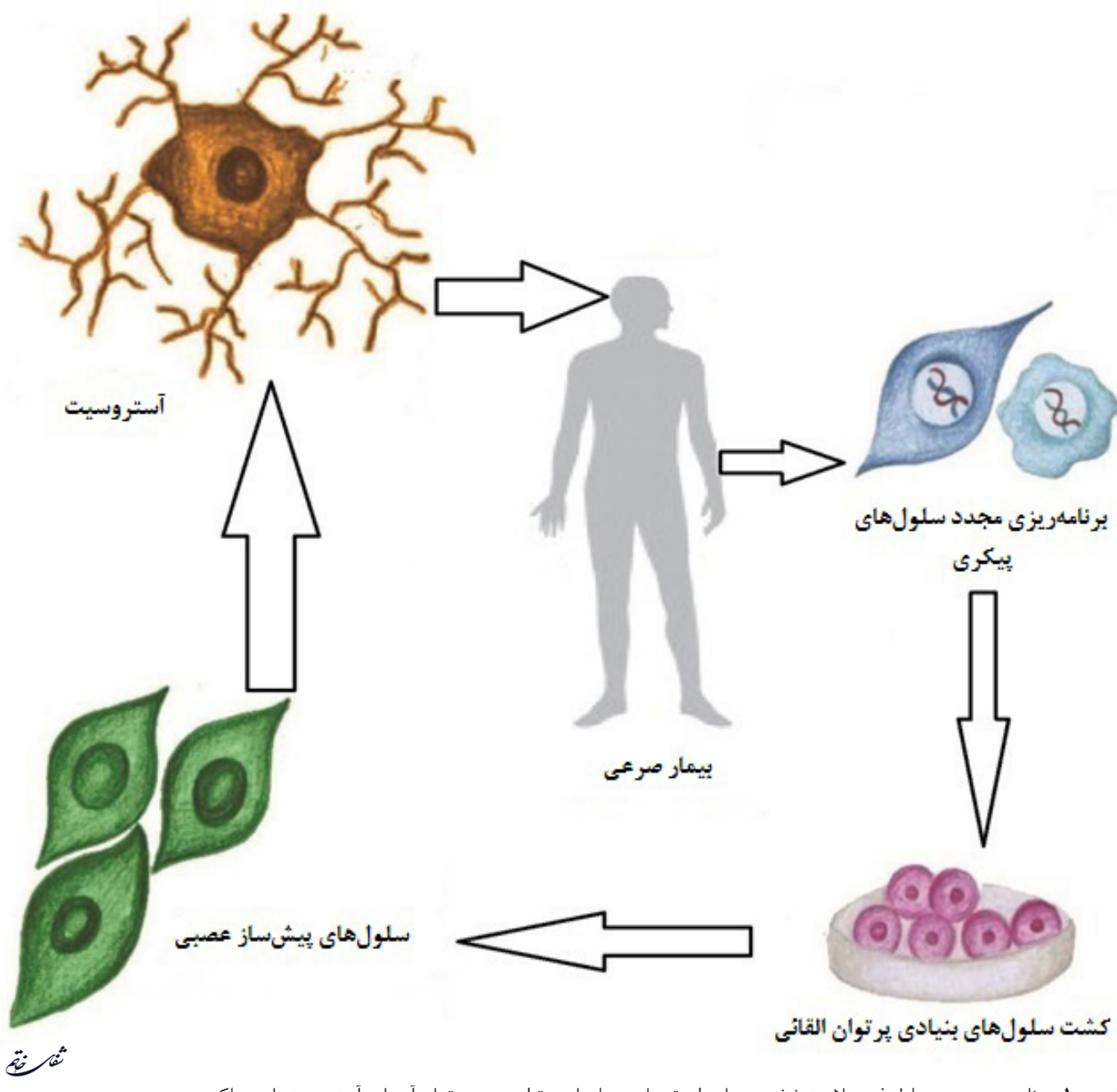

تصوير ا-برنامهريزى مجدد سلول فيبروبلاست شخص بيمار براى تبديل به سلوهاى يرتوان و سِّ تمايز آنها به آستروسيتهاى عملكردى.

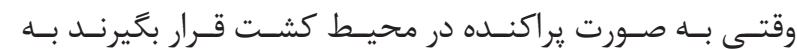

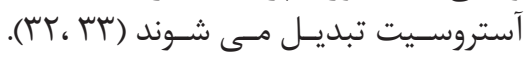

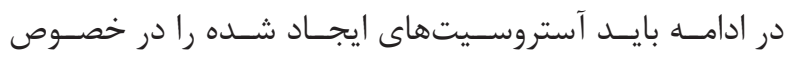

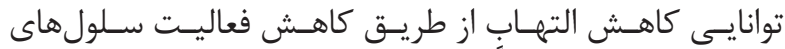

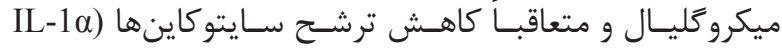
و (IL-1 ) ســنـيد.

بــه منظــور بررسـى ميـزان تمايـز سـلولها بـهـ آستروسـيت از

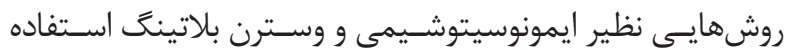

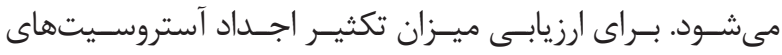

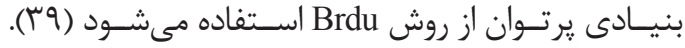

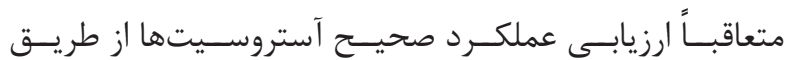

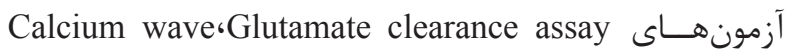

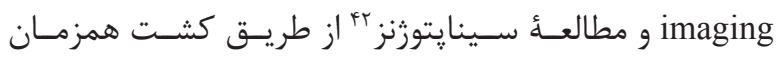

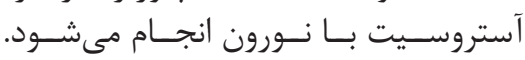

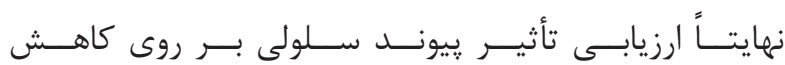

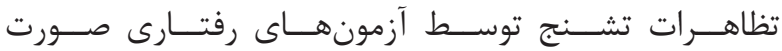

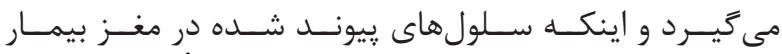

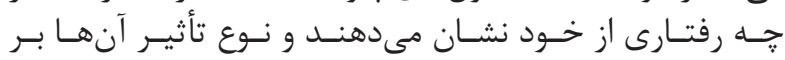

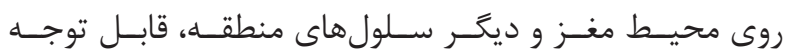

${ }^{42}$ Synaptogenesis 


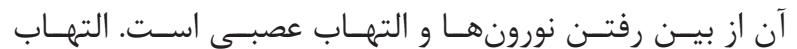

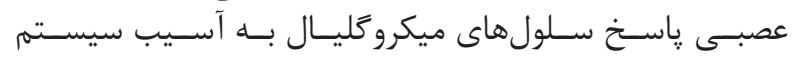

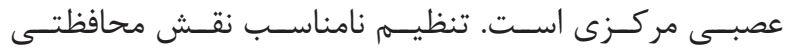

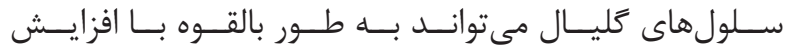

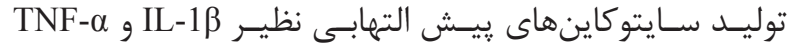

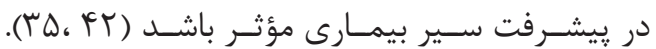

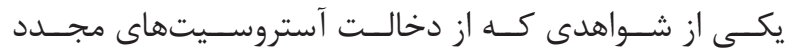

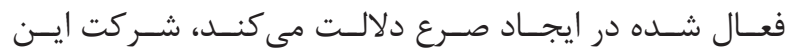

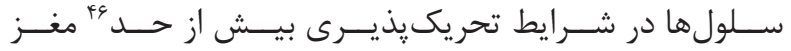

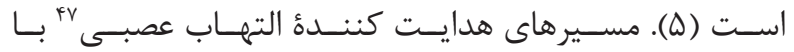

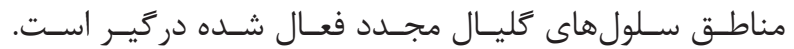

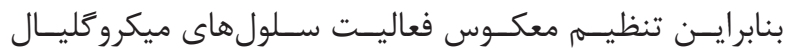

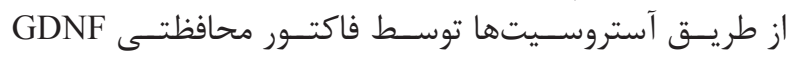

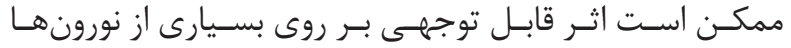
داشـته باشــد.

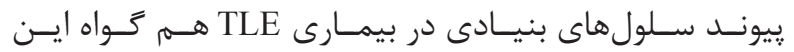

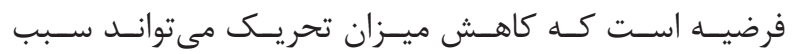

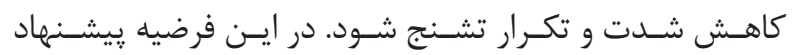

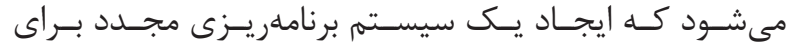

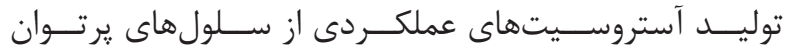

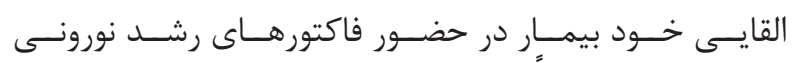

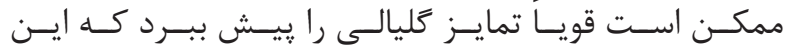

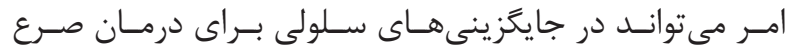

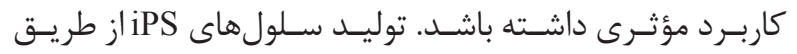

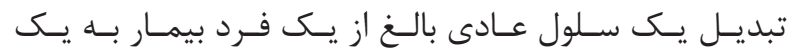

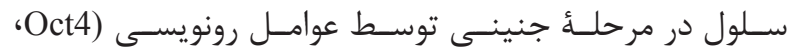

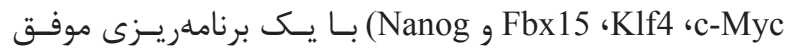

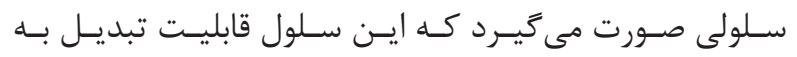

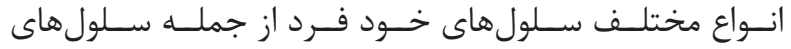
عصبـى و آستروســيتها را دارد.

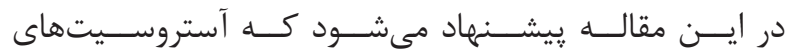

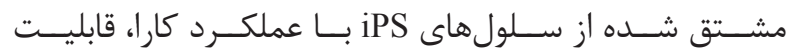

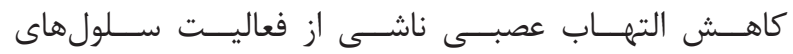

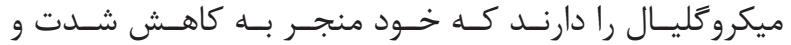

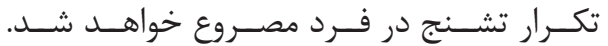

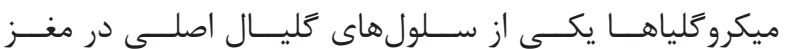

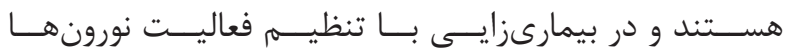

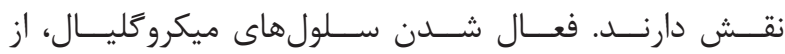

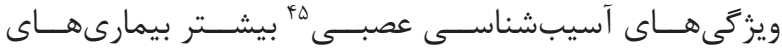

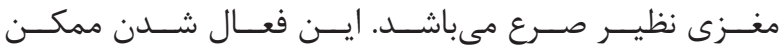

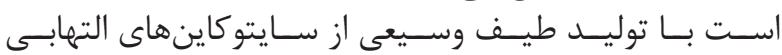

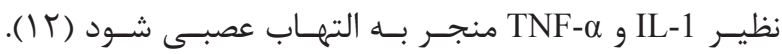

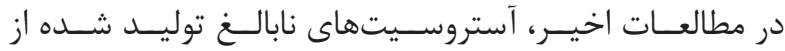

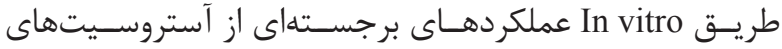

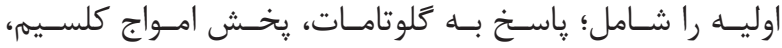

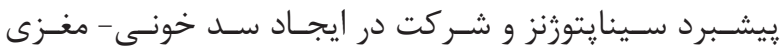

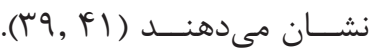

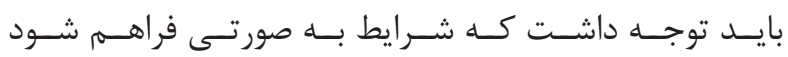

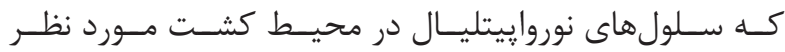

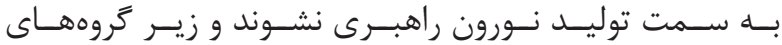

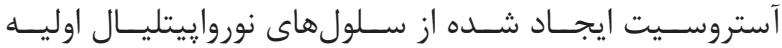

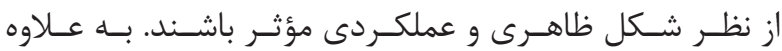

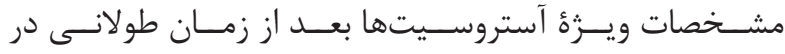

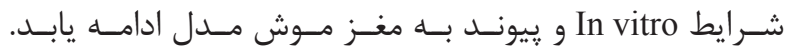

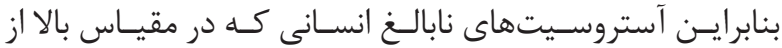

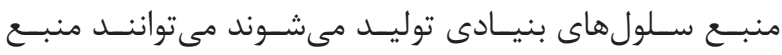

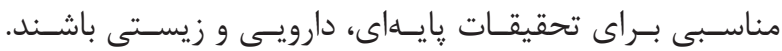

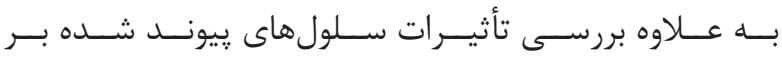

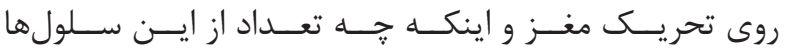

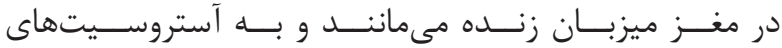

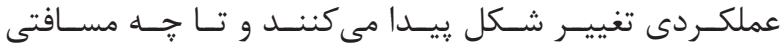

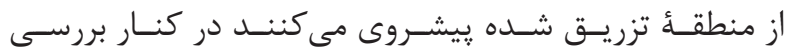

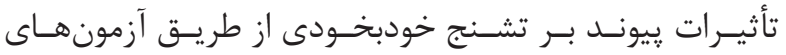

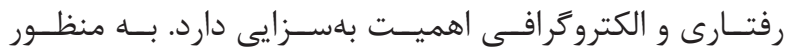

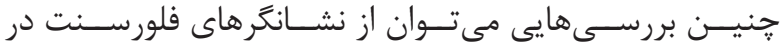

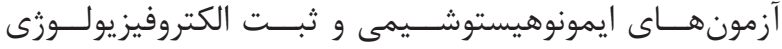

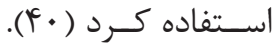

نتيجل

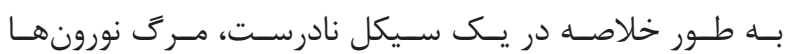

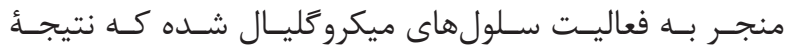

\footnotetext{
${ }^{45}$ Neuropathology

${ }^{46}$ Hyperexcitable Condition

${ }^{47}$ Neuroinflammatory Signaling Pathway
} 
1. Shetty AK. Progress in cell grafting therapy for temporal lobe epilepsy. Neurotherapeutics. 2011; 8(4): 721-35.

2. Gorji A, Straub H, Speckmann EJ. Epilepsy surgery: perioperative investigations of intractable epilepsy. Anat Embryol (Berl). 2005; 210(5-6): 525-37.

3. Seifert G, Carmignoto G, Steinhäuser C. Astrocyte dysfunction in epilepsy. Brain Res Rev. 2010; 63(1-2): 212-21.

4. McCarty MF. Down-regulation of microglial activation may represent a practical strategy for combating neurodegenerative disorders. Med Hypotheses. 2006; 67(2): 251-69.

5. Wetherington J, Serrano G, Dingledine R. Astrocytes in the epileptic brain. Neuron. 2008; 58(2): 168-78.

6. Mirrione MM, Tsirka SE. A functional role for microglia in epilepsy. Zaid Afawi. Clinical and Genetic Aspects of Epilepsy. $1^{\text {th }}$ ed. Croatia. 2011; p. 3-22.

7. Binder DK, Steinhäuser C. Functional changes in astroglial cells in epilepsy. Glia. 2006; 54(5): 358-68.

8. Contreras-Sesvold C. Reactive Astrocytes: Phenotypic and Functional Characteristics and Astrocytes as Neural Stem Cells. Master's thesis. Maryland. Uniformed Service University of the Health Sciences. Faculty of Neuroscience. 2006.

9. Ridet J, Privat A, Malhotra S, Gage F. Reactive astrocytes: cellular and molecular cues to biological function. Trends Neurosci. 1997; 20(12): 570-7.

10. Galou M, Colucci-Guyon E, Ensergueix D, Ridet JL, Gimenez y, Ribotta M, et al. Disrupted glial fibrillary acidic protein network in astrocytes from vimentin knockout mice. J Cell Biol. 1996; 133(4): 853-63.

11. Rinaldi R, Eliasson E, Swedmark S, Morgenstern R. Reactive intermediates and the dynamics of glutathione transferases. Drug Metab Dispos. 2002; 30(10): 1053-8.

12. Pascual O, Achour SB, Rostaing P, Triller A, Bessis A. Microglia activation triggers astrocyte-mediated modulation of excitatory neurotransmission. Proc Natl Acad Sci U S A. 2012; 109(4): E197-E205.

13. Vezzani A. Inflammation and epilepsy. Epilepsy Curr. 2005; 5(1): 1-6.
14. Block ML, Zecca L, Hong J-S. Microglia-mediated neurotoxicity: uncovering the molecular mechanisms. Nat Rev Neurosci. 2007; 8(1): 57-69.

15. Rocha SM, Cristovão AC, Campos FL, Fonseca CP, Baltazar G. Astrocyte-derived GDNF is a potent inhibitor of microglial activation. Neurobiol Dis. 2012; 47(3): 407-15.

16. Björklund A, Lindvall O. Cell replacement therapies for central nervous system disorders. Nat Neurosci. 2000; 3(6): 537-44.

17. Roper SN, Steindler DA. Stem cells as a potential therapy for epilepsy. Exp Neurol. 2013; 244: 59-66.

18. Muotri AR. Modeling epilepsy with pluripotent human cells. Epilepsy Behav. 2009; 14(1): 81-5.

19. Flake AW, Zanjani ED. In utero hematopoietic stem cell transplantation: ontogenic opportunities and biologic barriers. Blood. 1999; 94(7): 2179-91.

20. Strong M, Farrugia A, Rebulla P. Stem cell and cellular therapy developments. Biologicals. 2009; 37(2): 103-7.

21. Patel M, Yang S. Advances in reprogramming somatic cells to induced pluripotent stem cells. Stem Cell Rev. 2010; 6(3): 367-80.

22. Modarres Mousavi M, Ghaemi A, Ghadiri T, Mohammad Sadeghi S. Application of patient-specific induced Pluripotent Stem cells produced by somatic cells reprogramming for treatment of neurodegenerative diseases. Shefaye Khatam. 2013; 1(1): 19-23.

23. Robinton DA, Daley GQ. The promise of induced pluripotent stem cells in research and therapy. Nature. 2012; 481(7381): 295-305.

24. Park IH, Arora N, Huo H, Maherali N, Ahfeldt T, Shimamura A, et al. Disease-specific induced pluripotent stem cells. Cell. 2008; 134(5): 877-86.

25. Okita K, Nakagawa M, Hyenjong H, Ichisaka T, Yamanaka S. Generation of mouse induced pluripotent stem cells without viral vectors. Science. 2008; 322(5903): 949-53.

26. Shi Y, Desponts C, Do JT, Hahm HS, Schöler HR, Ding $\mathrm{S}$. Induction of pluripotent stem cells from mouse embryonic fibroblasts by Oct4 and Klf4 with smallmolecule compounds. Cell Stem Cell. 2008; 3(5): 568-74. 
27. Fulka J, First NL, Loi P, Moor RM. Cloning by somatic cell nuclear transfer. Bioessays. 1998; 20(10): 847-51.

28. Takahashi K, Tanabe K, Ohnuki M, Narita M, Ichisaka T, Tomoda K, et al. Induction of pluripotent stem cells from adult human fibroblasts by defined factors. Cell. 2007; 131(5): 861-72.

29. Naegele JR, Maisano X. Gene and stem cell therapies for treating epilepsy. Rho J, Sankar R, Stafstrom CE. Epilepsy: Mechanisms, Models, and Translational Perspectives (Neurological Disease and Therapy). $1^{\text {th }}$ ed. 2010 ; p. 583-601.

30. Tian GF, Azmi H, Takano T, Xu Q, Peng W, Lin J, et al. An astrocytic basis of epilepsy. Nat Med. 2005; 11(9): 973-81.

31. Berkovic SF, Mulley JC, Scheffer IE, Petrou S. Human epilepsies: interaction of genetic and acquired factors. Trends Neurosci. 2006; 29(7): 391-7.

32. Pfurtscheller G. Event-related synchronization (ERS): an electrophysiological correlate of cortical areas at rest. Electroencephalogr Clin Neurophysiol. 1992; 83(1): 62-9.

33. Sidhu KS. Induced pluripotent stem cells a slippery slope for neurodegenerative disease modeling. Open Stem Cell J. 2011; 3: 1-6.

34. Marchetto MC, Winner B, Gage FH. Pluripotent stem cells in neurodegenerative and neurodevelopmental diseases. Hum Mol Genet. 2010; 19(R1): R71-6.
35. Naegele JR, Maisano X, Yang J, Royston S, Ribeiro E. Recent advancements in stem cell and gene therapies for neurological disorders and intractable epilepsy. Neuropharmacol. 2010; 58(6): 855-64.

36. Buffo A, Rolando C, Ceruti S. Astrocytes in the damaged brain: molecular and cellular insights into their reactive response and healing potential. Biochem Pharmacol. 2010; 79(2): 77-89.

37. de Lanerolle NC, Lee TS, Spencer DD. Astrocytes and epilepsy. Neurotherapeutics. 2010; 7(4): 424-38.

38. Emdad L, D'Souza SL, Kothari HP, Qadeer ZA, Germano IM. Efficient differentiation of human embryonic and induced pluripotent stem cells into functionalastrocytes. StemCellsDev.2011;21(3):404-10.

39. Krencik R, Weick JP, Liu Y, Zhang ZJ, Zhang SC. Specification of transplantable astroglial subtypes from human pluripotent stem cells. Nat Biotechnol. 2011; 29(6): 528-34.

40. Sebe JY, Baraban SC. The promise of an interneuronbased cell therapy for epilepsy. Dev Neurobiol. 2011; 71(1): 107-17.

41. Krencik R, Zhang SC. Directed differentiation of functional astroglial subtypes from human pluripotent stem cells. Nat Protoc. 2011; 6(11): 1710-7.

42. Somera-Molina KC, Nair S, Van Eldik LJ, Watterson DM, Wainwright MS. Enhanced microglial activation and proinflammatory cytokine upregulation are linked to increased susceptibility to seizures and neurologic injury in a 'two-hit'seizure model. Brain Res. 2009; 1282: $162-72$. 